\title{
UNIFORMLY STABLE MIXED HP-FINITE ELEMENTS ON MULTILEVEL ADAPTIVE GRIDS WITH HANGING NODES
}

\author{
FRIEDHELM SCHIEWECK ${ }^{1}$
}

\begin{abstract}
We consider a family of quadrilateral or hexahedral mixed $h p$-finite elements for an incompressible flow problem with $Q_{r}$-elements for the velocity and discontinuous $P_{r-1}$-elements for the pressure where the order $r$ can vary from element to element between 2 and an arbitrary bound. For multilevel adaptive grids with hanging nodes and a sufficiently small mesh size, we prove the inf-sup condition uniformly with respect to the mesh size and the polynomial degree.
\end{abstract}

Mathematics Subject Classification. 65N30, 65N35, 76D07.

Received January 10, 2007. Revised October 2, 2007.

Published online April 1st, 2008.

\section{INTRODUCTION}

For the numerical solution of incompressible flow problems, the mixed $h p$-finite element method is a well established efficient technique (see [22] and the references therein). It is well known that the inf-sup condition has to be satisfied in order to guarantee stability and accuracy of the discrete solution [9]. The inf-sup condition has been proven for many pairs of finite element spaces for the $h$-version (see e.g. $[5,8,9,11,16,20,23]$ ) as well as for the $p$ - and $h p$-version (see e.g. $[1,4,10,21,24,25]$ ). In the above-mentioned papers on the $h$ - and $h p$-version, it has been shown that the inf-sup constant is independent of the mesh size $h$. However, in the literature on the $h$-version (see e.g. $[8,11,16,20,23]$ ), the dependence of the inf-sup constant on the polynomial degree has not been investigated.

For the $h p$-version, the inf-sup condition was proved in [24] for different families of finite element pairs, in particular for the pairs $\left(\mathbb{Q}_{r}, \mathbb{P}_{r-1}^{\text {disc }}\right)$ and $\left(\mathbb{Q}_{r+1}, \mathbb{Q}_{r-1}^{\text {disc }}\right)$ where "disc" means that the space consists of discontinuous functions which are elementwise defined by polynomials. It was proved that the inf-sup constant decreases as $C r^{-(d-1) / 2}$ where $d$ denotes the dimension of the domain and $C$ a constant independent of the mesh size $h$ and the polynomial degree $r$. In [21,25], for the two and three dimensional case, respectively, the inf-sup condition was proved for the family of finite element pairs $\left(\mathbb{Q}_{r}, \mathbb{Q}_{r-2}^{\text {disc }}\right)$ on so-called anisotropically and geometrically refined meshes with hanging nodes. It has been shown that the inf-sup constant can be estimated from below by $\mathrm{Cr}^{-1 / 2}$ in the 2D-case and $C r^{-3 / 2}$ in the 3D-case where the constant $C$ is independent of the mesh size $h$, the polynomial degree $r$ and the element aspect ratio. In [21,24,25], the cells of the mesh are assumed to be affine equivalent to a reference element. Therefore, the analysis covers only the case where the quadrilateral or

\footnotetext{
Keywords and phrases. Stokes problem, inf-sup condition, mixed $h p$-FEM, quadrilateral and hexahedral finite elements, multilevel adaptive grids, hanging nodes.

1 Institut für Analysis und Numerik, Otto-von-Guericke-Universität Magdeburg, Postfach 4120, 39016 Magdeburg, Germany. schiewec@ovgu.de
} 
hexahedral elements are parallelograms or parallelepipeds, respectively, which seems to be too restrictive from the practical point of view.

For two dimensional non-affine equivalent quadrilateral meshes with hanging nodes, Ainsworth and Coggins [1] proposed a family of finite element pairs $\left(\widetilde{\mathbb{Q}}_{r}, \mathbb{Q}_{r-1}^{\prime}\right)$ which is inf-sup stable uniformly with respect to the mesh size $h$ and the polynomial degree $r$. Here $\mathbb{Q}_{r-1}^{\prime}$ denotes a continuous pressure space constructed from the discontinuous space $\mathbb{P}_{r}^{\text {disc }}$ by some additional $\mathbb{Q}_{r-1}$-functions. The velocity space $\widetilde{\mathbb{Q}}_{r}$ denotes a modification of the usual space $\mathbb{Q}_{r}$ where the basis functions associated with nodes at the element boundary are defined by means of the usual reference transformation but the functions corresponding to interior element nodes are based on the Piola transformation (see [10] for a similar construction). This causes some extra difficulties in the implementation, in particular for the derivatives of the velocity functions.

In this paper, we prove, for the $d$-dimensional case, $d \in\{2,3\}$, a uniform inf-sup condition for the family of finite element pairs $\left(\mathbb{Q}_{r}, \mathbb{P}_{r-1}^{\text {disc }}\right)$ on so called multilevel adaptive grids of quadrilateral or hexahedral elements with hanging nodes. By $\mathbb{P}_{r-1}^{\text {disc }}$ we denote the space of mapped discontinuous functions which are defined on the reference element by polynomials of total degree not greater than $r-1$. Note that this mapped space may have non-optimal approximation properties on general quadrilateral or hexahedral grids $[2,5]$. However, one can show that it has optimal approximation properties on multilevel adaptive grids $[17,19]$. Multilevel adaptive grids are created from a regular coarse grid by means of recursive refinement of existing elements into $2^{d}$ many son-elements combined with the introduction of hanging nodes. From the practical point of view, such grids occur very naturally in applications with adaptive refinement based on a posteriori error estimators (see e.g. $[13,14])$. Typical grids are depicted in Figure 1. Note that we do not assume that the elements are affine equivalent.

The new contribution of this paper compared to the results of $[16,20,24]$ on the element pair $\left(\mathbb{Q}_{r}, \mathbb{P}_{r-1}^{\text {disc }}\right)$ is that we prove the inf-sup constant to be independent of the polynomial degree $r$ if the mesh size $h$ is sufficiently small. We use the result of Bernardi and Maday [4] that, for the situation of just one element $K=(-1,1)^{d}$, the inf-sup constant of this element pair is independent of the polynomial degree $r$. The main idea is to use a new modified Piola transformation to transform the inf-sup condition from the reference element to the original element. This proves the local inf-sup condition. The proof of the global inf-sup condition is based on the technique of Boland and Nicolaides [6] and an result in [16] for low order subspaces. Note that we assume shape-regular grids with possibly hanging nodes and that we do not consider anisotropic mesh refinements.

As a model for an incompressible flow problem we consider the Stokes equations with homogeneous Dirichlet conditions: Find a velocity $u$ and a pressure $p$ such that

$$
\begin{aligned}
-\nu \Delta u+\nabla p & =f & & \text { in } \Omega, \\
\operatorname{div} u & =0 & & \text { in } \Omega, \\
u & =0 & & \text { on } \Gamma .
\end{aligned}
$$

Here, $\Omega$ denotes a bounded domain in $\mathbb{R}^{d}, d \in\{2,3\}$, with a polygonal or polyhedral boundary $\Gamma, \nu>0$ the kinematic viscosity and $f$ a given body force. Then, a pair $\left(X^{N}, M^{N}\right)$ of conforming finite element spaces for the approximation of the velocity $u \in\left(H_{0}^{1}(\Omega)\right)^{d}$ and the pressure $p \in L_{0}^{2}(\Omega)$ is said to fulfill the discrete inf-sup condition if the following estimate holds:

$$
\exists \beta>0: \quad \inf _{q^{N} \in M^{N} \backslash\{0\}} \sup _{v^{N} \in X^{N} \backslash\{0\}} \frac{\left(\operatorname{div} v^{N}, q^{N}\right)}{\left|v^{N}\right|_{1, \Omega}\left\|q^{N}\right\|_{0, \Omega}} \geq \beta .
$$

For the stability and accuracy of the solution $\left(u^{N}, p^{N}\right) \in X^{N} \times M^{N}$ of the discrete Stokes problem, it is desirable that the inf-sup constant $\beta$ in (0.2) is independent of the mesh size and the polynomial degree. Note that the same requirement arises for solving the incompressible Navier-Stokes equations.

The outline of this paper is as follows. In Section 1, we introduce the notations, the concept of multilevel adaptive grids and the used finite element spaces. In Section 2.1, we present the global and local inf-sup conditions that are used for the technique of Boland and Nicolaides. The modified Piola transformation is 

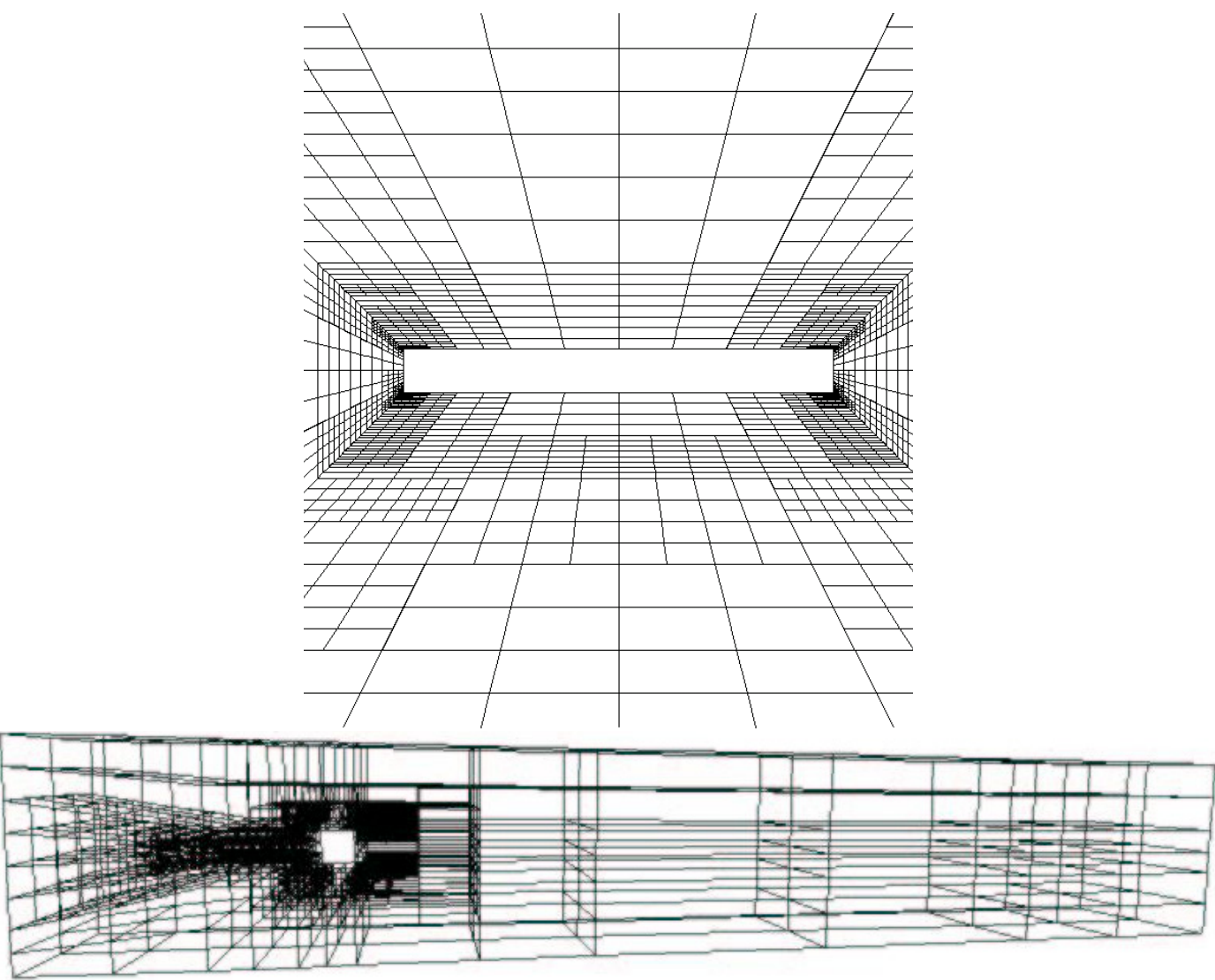

Hivision 2.0

Figure 1. Typical local refined grids with hanging nodes; (top) two-dimensional grid for the computation of an exterior flow problem [7]; (bottom) three-dimensional grid for a channel flow problem [12].

introduced and studied in Section 2.2. In Section 2.3, we prove the local inf-sup condition. Finally, we summarize our main result in Section 2.4.

\section{PRELIMINARIES AND NOTATIONS}

\subsection{General notations}

Let $\Omega \subset \mathbb{R}^{d}, d \in\{2,3\}$, be a bounded domain with a polygonal or polyhedral boundary. For a set $G \subset \Omega$, let $|\cdot|_{m, p, G}$ and $\|\cdot\|_{m, p, G}$ denote the usual semi-norm and norm in the Sobolev spaces $W^{m, p}(G)$ and $\left(W^{m, p}(G)\right)^{d}$, respectively. For the Hilbert spaces $H^{m}(G)$ and $\left(H^{m}(G)\right)^{d}$, we omit the index $p$ and denote the semi-norm and norm by $|\cdot|_{m, G}$ and $\|\cdot\|_{m, G}$. The scalar product in $L^{2}(G)$ and $\left(L^{2}(G)\right)^{d}$ will be denoted by $(\cdot, \cdot)_{G}$. Let $L_{0}^{2}(G) \subset L^{2}(G)$ be the subspace of functions with an integral mean value of zero. By $\mathbb{P}_{m}(G)$ we denote the space of all polynomials on the domain $G \subset \mathbb{R}^{d}$ with total degree less than or equal to $m$ and by $\mathbb{Q}_{m}(G)$ the space of those polynomials where the maximum power in each coordinate is less than or equal to $m$. For $p \in[1, \infty]$, both the usual $p$-norm of a vector in $\mathbb{R}^{d}$ and the induced $p$-norm of a $d \times d$-matrix are denoted by $\|\cdot\|_{p}$. For a set $G \subset \mathbb{R}^{d}$, we denote by $\operatorname{int}(G)$ and $\bar{G}$ the interior and closure of $G$, respectively. Throughout this paper, $C$ will denote a generic constant which may have different values at different places. All these constants 
occurring inside of any estimate will be - unless it is explicitly stated - independent of the local and global mesh parameters $h_{K}$ and $h$ and the polynomial degree vector $\underline{r}$ which will be defined below.

\subsection{Multilevel adaptive grids}

Let the bounded domain $\Omega \subset \mathbb{R}^{d}$ be partitioned by a grid $\mathcal{T}_{h}$ consisting of elements $K \in \mathcal{T}_{h}$ which are assumed to be open quadrilaterals in the 2D-case and open hexahedrons in the 3D-case such that $\bar{\Omega}=\cup_{K \in \mathcal{T}_{h}} \bar{K}$. For an element $K \in \mathcal{T}_{h}$, we denote by $h_{K}$ the diameter of the element $K$. The mesh size $h$ of $\mathcal{T}_{h}$ is given by $h:=\max _{K \in \mathcal{T}_{h}} h_{K}$. We denote by $F_{K}: \widehat{K} \rightarrow K$ the mapping between the reference element $\widehat{K}:=(-1,+1)^{d}$ and the original element $K$. In the sequel of this paper, the mapping $F_{K}$ is assumed to be multi-linear, i.e. $F_{K} \in\left(\mathbb{Q}_{1}(\widehat{K})\right)^{d}$. Therefore, the two dimensional faces of $3 \mathrm{D}$ hexahedral elements $K \in \mathcal{T}_{h}$ can be curved in general.

For our analysis, we assume that the mesh family $\left\{\mathcal{T}_{h}\right\}$ is shape-regular in the sense presented in [20], see also $[16,19]$. Let us recall the definition. By a Taylor expansion of $F_{K}(\hat{x})$ at the point $\hat{x}=0$ we get

$$
F_{K}(\hat{x})=F_{K}(0)+B_{K} \hat{x}+G_{K}(\hat{x}),
$$

with $B_{K}:=D F_{K}(0)$ and $G_{K}(\hat{x}):=F_{K}(\hat{x})-F_{K}(0)-B_{K} \hat{x}$. We denote by $\widehat{S} \subset \widehat{K}$ the $d$-simplex with the vertices $0, e_{1}, \ldots, e_{d}$, where $e_{i} \in \mathbb{R}^{d}$ denotes the $i$-th unit vector. By $S_{K}$ we denote the $d$-simplex which is the image of $\widehat{S}$ under the affine mapping $\hat{x} \mapsto B_{K} \hat{x}+F_{K}(0)$. We assume that the simplices $S_{K}$ satisfy the usual shape regularity condition

$$
\frac{h_{S_{K}}}{\rho_{S_{K}}} \leq C_{s} \quad \forall K \in \mathcal{T}_{h},
$$

where $h_{S_{K}}$ denotes the diameter of $S_{K}$ and $\rho_{S_{K}}$ the diameter of the largest ball that can be inscribed into $S_{K}$. For an element $K \in \mathcal{T}_{h}$ and $p \in[1, \infty]$, we define the quantity $\gamma_{p}(K)$ by

$$
\gamma_{p}(K):=\sup _{\hat{x} \in \widehat{K}}\left\|B_{K}^{-1} D F_{K}(\hat{x})-I\right\|_{p} .
$$

$\gamma_{p}(K)$ is a measure of the deviation of $K$ from a parallelogram or a parallelepiped, respectively. Note that $\gamma_{p}(K)=0$ is equivalent to the fact that the mapping $F_{K}$ is affine.

Definition 1. A family $\left\{\mathcal{T}_{h}\right\}$ of meshes consisting of quadrilateral or hexahedral elements is called shaperegular if there exist a fixed $p \in[1, \infty]$ and $h$-independent constants $C_{s}$ and $\gamma_{0}$ such that $(1.2)$ and the estimates

are satisfied.

$$
\gamma_{p}(K) \leq \gamma_{0}<1 \quad \forall K \in \mathcal{T}_{h}
$$

It has been proven in [20] that for a family $\left\{\mathcal{T}_{h}\right\}$ of meshes, which is shape-regular in the sense of Definition 1 , the reference mappings $F_{K}: \widehat{K} \rightarrow K$ are bijective for all elements $K \in \mathcal{T}_{h}$. Furthermore, one can find sufficient conditions in [20], Section 2, that guarantee the fulfillment of the assumption (1.4). These conditions can be easily checked in practical computations. For example, in the 2D-case, the condition $\gamma_{1}(K)<1$ is equivalent to the fact that the quadrilateral element $K$ is convex. Our assumption that the meshes are shape-regular in the sense of Definition 1 guarantees that the reference mapping $F_{K}: \widehat{K} \rightarrow K$ satisfies for all $K \in \mathcal{T}_{h}$ the following properties (for the proof, see [15]):

$$
\begin{gathered}
\left\|D F_{K}(\hat{x})\right\|_{\infty} \leq C h_{K} \quad \forall \hat{x} \in \widehat{K}, \\
C h_{K}^{d} \leq\left|\operatorname{det}\left(D F_{K}(\hat{x})\right)\right| \leq C^{\prime} h_{K}^{d} \quad \forall \hat{x} \in \widehat{K} .
\end{gathered}
$$

In this paper, we study the case of hanging nodes, i.e., the usual assumption of a regular grid $\mathcal{T}_{h}$ has to be weakened. In the following, we will describe the type of grids that are treated in this paper. We assume that 


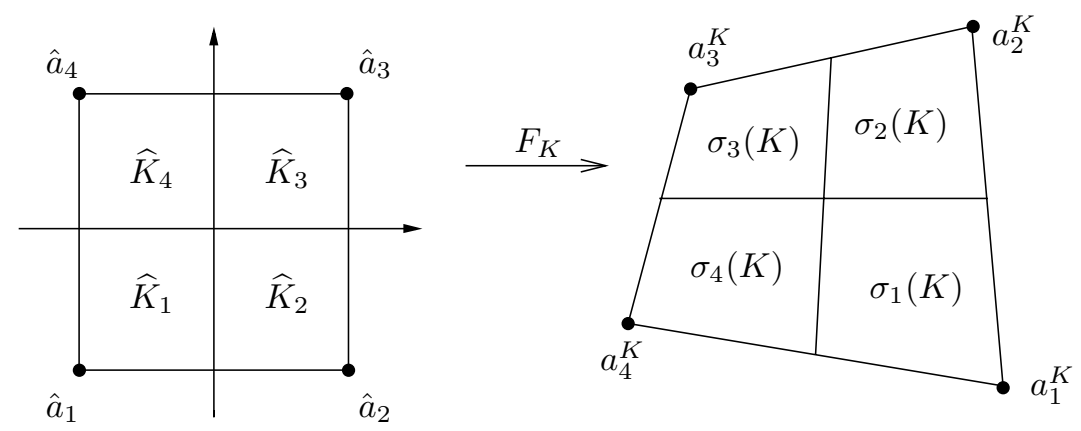

Figure 2. Refinement of element $K$ into son-elements $\sigma_{i}(K), i=1, \ldots, 2^{d}$.

$\mathcal{T}_{h}$ is a multilevel adaptive grid generated by a refinement process in the following way. We start with an initial partition $\mathcal{T}^{0}$ of the domain $\Omega$ into elements $K \in \mathcal{T}^{0}$ of grid-level 0, i.e. $\Omega=\operatorname{int}\left(\bigcup_{K \in \mathcal{T}^{0}} \bar{K}\right)$. The grid $\mathcal{T}^{0}$ is assumed to be regular in the usual sense, i.e., for any two different elements $K_{1}, K_{2} \in \mathcal{T}^{0}$ the intersection $\overline{K_{1}} \cap \overline{K_{2}}$ is either empty or a common $(d-m)$-dimensional face of $K_{1}$ and $K_{2}$ where $m \in\{1, \ldots, d\}$. Now, starting with the elements $K \in \mathcal{T}^{0}$, an existing element $K$ can be refined, i.e., it can be split into $2^{d}$ many new elements called son-elements which are denoted by $\sigma_{i}(K), i=1, \ldots, 2^{d}$, see Figure 2 . These son-elements are defined as follows. Let $\widehat{K}_{i}, i \in\left\{1, \ldots, 2^{d}\right\}$, be the son-elements of the reference element $\widehat{K}=(-1,1)^{d}$ which are congruent $d$-cubes with $\left|\widehat{K}_{i}\right|=1$. Then, the $i$-th son-element $\sigma_{i}(K)$ of the original element $K$ is defined as $\sigma_{i}(K):=F_{K}\left(\widehat{K}_{i}\right)$ for all $i=1, \ldots, 2^{d}$. Note that in the 3D-case, even if an element $K$ has only planar faces, it can happen that the son-elements $\sigma_{i}(K)$ have curved faces in the interior of $K$ (see [18], Rem. 3.3, for an example). For a new element $K^{\prime}=\sigma_{i}(K)$, we will say that $K$ is the father-element of $K^{\prime}$ and we will write $K=\mathcal{F}\left(K^{\prime}\right)$. If an element $K$ is refined then it is replaced in the partition of the domain $\Omega$ by the set of its son-elements $\sigma_{i}(K), i=1, \ldots, 2^{d}$. The new elements can be refined repeatedly until the final partition $\mathcal{T}_{h}$ of $\Omega$ is created. Examples of such grids used in practical computations can be found in [13,14].

Definition 2. For an element $K \in \mathcal{T}_{h}$, generated from the initial grid $\mathcal{T}^{0}$ by the refinement process described above, we define the refinement level $\ell(K)$ as $\ell(K):=0$ if $K \in \mathcal{T}^{0}$ and $\ell(K):=m \geq 1$ if there exists a chain of $m$ father-elements $K_{i}, i=m-1, \ldots, 0$, starting from $K_{m}:=K$ and defined by $K_{i-1}:=\mathcal{F}\left(K_{i}\right)$ for $i=m, \ldots, 1$, such that $K_{0} \in \mathcal{T}^{0}$. The grid $\mathcal{T}_{h}$, generated by the refinement process defined above, is called 1-irregular if the condition

$$
\left|\ell(K)-\ell\left(K^{\prime}\right)\right| \leq 1
$$

holds for any pair of different adjacent elements $K, K^{\prime} \in \mathcal{T}_{h}$ where $\partial K \cap \partial K^{\prime}$ is a one- or two-dimensional manifold.

Note that, in the 3D-case, the condition (1.7) has to be satisfied if the elements $K$ and $K^{\prime}$ have a common edge or a common face. The refinement level $\ell(K)$ is equal to the number of refinement steps that are needed to generate the element $K$ from an element of the coarsest grid $\mathcal{T}^{0}$.

Our concept of 1-irregular grids is a special case of the concept of K-meshes introduced by Babuška and Miller [3]. In many practical computations, 1-irregular grids are used. They are sufficient to describe complicated structures in a reasonable way, see $e . g$. [13,14]. An example that shows the capabilities of 1-irregular quadrilateral grids to resolve an inner fine scale structure is presented in Figure 3.

An advantage of multilevel adaptive grids is that the reference mapping $F_{K}$ behaves - up to a power of the mesh size $h$ - like an affine mapping. This is one of the key points of the analysis in this paper. We have the following result. 


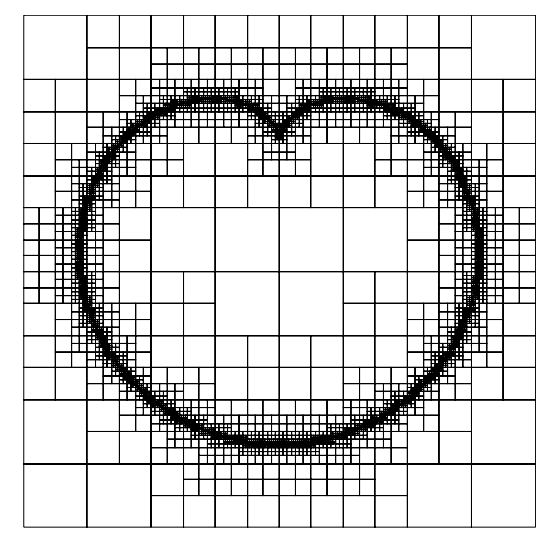

FiguRE 3. Resolution of an inner fine scale structure by a multilevel adaptive 1-irregular grid.

Lemma 3. Let $\left\{\mathcal{T}_{h}\right\}$ be a family of multilevel adaptive grids generated from a shape-regular coarse grid $\mathcal{T}^{0}$ as described above. Then, there exist constants $C_{1}\left(m, \mathcal{T}^{0}\right)$ and $C_{2}\left(\mathcal{T}^{0}\right)$ such that the following estimates are satisfied for all $K \in \mathcal{T}_{h}$

$$
\begin{aligned}
\left|F_{K}\right|_{m, \infty, \widehat{K}} & \leq C_{1}\left(m, \mathcal{T}^{0}\right) h_{K}^{m} \quad \forall m \in\{0,1,2,3\}, \\
\left\|J_{K}(\widehat{x})-B_{K}\right\|_{p} & \leq C_{2}\left(\mathcal{T}^{0}\right) h_{K}^{2} \quad \forall p \in[1, \infty], \quad \forall \widehat{x} \in \widehat{K},
\end{aligned}
$$

where $J_{K}(\widehat{x}):=D F_{K}(\widehat{x})$ and $B_{K}:=D F_{K}(0)$.

Proof. See Lemma 5 in [19].

\subsection{Finite element spaces}

Let $\underline{r}$ denote the degree vector $\underline{r}:=\left\{r_{K}: K \in \mathcal{T}_{h}\right\}$ which contains the polynomial degrees $r_{K}$ of the velocity approximation on each element $K$. In this paper, we assume that

$$
r_{K} \geq 2 \quad \forall K \in \mathcal{T}_{h}
$$

The finite element space of the velocity $X^{N} \subset\left(H_{0}^{1}(\Omega)\right)^{d}$ associated with the grid $\mathcal{T}_{h}$ and the degree vector $\underline{r}$ is defined as $X^{N}:=\left(S^{N}\right)^{d}$ with the scalar finite element space

$$
S^{N}:=S\left(\Omega ; \underline{r}, \mathcal{T}_{h}\right):=\left\{\phi \in H_{0}^{1}(\Omega):\left.\phi\right|_{K} \circ F_{K} \in \mathbb{Q}_{r_{K}}(\widehat{K}) \quad \forall K \in \mathcal{T}_{h}\right\}
$$

The finite element space $M^{N} \subset L_{0}^{2}(\Omega)$ for the approximation of the pressure is chosen as the following mapped space

$$
M^{N}:=M\left(\Omega ; \underline{r}, \mathcal{T}_{h}\right):=\left\{q \in L_{0}^{2}(\Omega):\left.q\right|_{K} \circ F_{K} \in \mathbb{P}_{r_{K}-1}(\widehat{K}) \quad \forall K \in \mathcal{T}_{h}\right\} .
$$

Then, the discrete Stokes problem reads: Find $\left(u^{N}, p^{N}\right) \in X^{N} \times M^{N}$ such that

$$
\begin{aligned}
& \nu\left(\nabla u^{N}, \nabla v^{N}\right)_{\Omega}-\left(p^{N}, \nabla \cdot v^{N}\right)_{\Omega}=\left(f, v^{N}\right)_{\Omega} \quad \forall v^{N} \in X^{N} \\
& \left(q^{N}, \nabla \cdot u^{N}\right)_{\Omega}=0 \quad \forall q^{N} \in M^{N} .
\end{aligned}
$$

For the subsequent analysis we need also the finite element space for the velocity with the polynomial order of 2, i.e. $X_{h}^{2}:=\left(S_{h}^{2}\right)^{d}$ based on the scalar finite element space

$$
S_{h}^{2}:=\left\{\phi \in H_{0}^{1}(\Omega):\left.\phi\right|_{K} \circ F_{K} \in \mathbb{Q}_{2}(\widehat{K}) \forall K \in \mathcal{T}_{h}\right\}
$$


as well as the finite element space of the piecewise constant pressure functions

$$
M_{h}^{0}:=\left\{q \in L_{0}^{2}(\Omega):\left.q\right|_{K} \in \mathbb{P}_{0}(K) \quad \forall K \in \mathcal{T}_{h}\right\} .
$$

Some attention is required to ensure interelement continuity in (1.11) and (1.14) in the case of hanging nodes or if $r_{K}$ is different on two adjacent cells of an inner element face. We refer to [22] for a detailed treatment of this issue. Note that, due to the assumption (1.10), it holds $X_{h}^{2} \subset X^{N}$.

\section{PROVING THE INF-SUP CONDITION}

\subsection{Global and local inf-sup conditions}

Our approach to prove the inf-sup condition (0.2) is based on the technique proposed by Boland and Nicolaides [6] (see also [11], Sect. II.1.4). For the partitioning of the domain $\Omega$ into non-overlapping subdomains, we take the elements $K \in \mathcal{T}_{h}$. According to the finite element spaces $X^{N}=\left(S^{N}\right)^{d}$ and $M^{N}$ defined in (1.11) and (1.12), respectively, we define for each element $K \in \mathcal{T}_{h}$ the following local spaces

$$
\begin{aligned}
& X^{N}(K):=\left\{v \in\left(H_{0}^{1}(K)\right)^{d}: v \circ F_{K} \in\left(\mathbb{Q}_{r_{K}}(\widehat{K})\right)^{d}\right\}, \\
& M^{N}(K):=\left\{q \in L_{0}^{2}(K): q \circ F_{K} \in \mathbb{P}_{r_{K}-1}(\widehat{K})\right\} .
\end{aligned}
$$

We say that the finite element spaces $X^{N}$ and $M^{N}$ fulfill the local inf-sup condition uniformly with respect to the mesh size $h$ and the polynomial degree vector $\underline{r}$ if there exists a constant $\beta_{0}>0$, such that it holds

$$
\inf _{q^{N} \in M^{N}(K) \backslash\{0\}} \sup _{v^{N} \in X^{N}(K) \backslash\{0\}} \frac{\left(\nabla \cdot v^{N}, q^{N}\right)_{K}}{\left|v^{N}\right|_{1, K}\left\|q^{N}\right\|_{0, K}} \geq \beta_{0}, \quad \forall K \in \mathcal{T}_{h} .
$$

Now, in order to prove the global inf-sup condition for our finite element spaces $X^{N}=\left(S^{N}\right)^{d}$ and $M^{N}$ we only have to verify the local inf-sup condition (2.3) and the following global inf-sup condition for a suitable pair of low order subspaces $\left(\bar{X}^{N}, M_{h}^{0}\right)$.

Lemma 4. Let the local inf-sup condition (2.3) be fulfilled uniformly with respect to the mesh size $h$ and the degree vector $\underline{\underline{r}}$. Furthermore, let there exist a subspace $\bar{X}^{N} \subset X^{N}$ such that the pair of finite element spaces $\left(\bar{X}^{N}, M_{h}^{0}\right)$ fulfills the global inf-sup condition

$$
\inf _{q_{h} \in M_{h}^{0} \backslash\{0\}} \sup _{v_{h} \in \bar{X}^{N} \backslash\{0\}} \frac{\left(\nabla \cdot v_{h}, q_{h}\right)_{\Omega}}{\left|v_{h}\right|_{1, \Omega}\left\|q_{h}\right\|_{0, \Omega}} \geq \bar{\beta},
$$

with a constant $\bar{\beta}>0$ independent of $h$ and $\underline{r}$. Then, the pair $\left(X^{N}, M^{N}\right)$ satisfies the global inf-sup condition (0.2) with a constant $\beta>0$ which depends on $\bar{\beta}$ and $\beta_{0}$ but not on $h$ and $\underline{r}$.

Proof. See Theorem II.1.12 in [11].

The global inf-sup condition (2.4) of the pair of spaces $\left(\bar{X}^{N}, M_{h}^{0}\right)$ can be proved for the second order subspace $\bar{X}^{N}=X_{h}^{2} \subset X^{N}$.

Lemma 5. Let $\mathcal{T}_{h}$ be a multilevel adaptive grid, which is assumed to be 1-irregular and shape-regular. Then, the pair of finite element spaces $\left(\bar{X}^{N}, M_{h}^{0}\right)$ with $\bar{X}^{N}:=X_{h}^{2}$ satisfies the global inf-sup condition (2.4) with a constant $\bar{\beta}>0$ independent of $h$ and $\underline{r}$.

Proof. See [16], Lemma 9. 


\subsection{Modified Piola transformation}

The Piola transformation is a well known tool to establish the local inf-sup condition $[1,10]$. For an element $K \in \mathcal{T}_{h}$ and a given function $\widehat{v} \in\left(H^{1}(\widehat{K})\right)^{d}$, the Piola transformation $\mathcal{P}_{K} \widehat{v} \in\left(H^{1}(K)\right)^{d}$ is defined by

$$
\left(\mathcal{P}_{K} \widehat{v}\right)(x):=\frac{1}{\operatorname{det}\left(J_{K}(\widehat{x})\right)} J_{K}(\widehat{x}) \widehat{v}(\widehat{x}) \quad \forall x \in K,
$$

where $J_{K}(\widehat{x}):=D F_{K}(\widehat{x})$ and $\widehat{x}:=F_{K}^{-1}(x)$. Due to the property (1.6) of grids which are shape-regular in the sense of Definition 1, the expression in (2.5) is well defined. The Piola transformation has the following essential property.

Lemma 6. For a given element $K \in \mathcal{T}_{h}$ and functions $\widehat{v} \in\left(H_{0}^{1}(\widehat{K})\right)^{d}$ and $\widehat{q} \in H^{1}(\widehat{K})$, let $v \in\left(H_{0}^{1}(K)\right)^{d}$ and $q \in H^{1}(K)$, be the associated functions $v:=\mathcal{P}_{K} \widehat{v}$ and $q:=\widehat{q} \circ F_{K}^{-1}$, respectively. Then, it holds

$$
(\operatorname{div} v, q)_{K}=(\widehat{\operatorname{div}} \widehat{v}, \widehat{q})_{\widehat{K}} .
$$

Proof. We easily obtain (2.6) if we apply partial integration and transform the integral over $K$ into an integral over $\widehat{K}$, see [10].

For our local velocity space $X^{N}(K)$ defined in (2.1), a problem in the proof of the local inf-sup condition is the fact that for a given function $\widehat{v} \in\left(\mathbb{Q}_{r_{K}}(\widehat{K}) \cap H_{0}^{1}(\widehat{K})\right)^{d}$ the transformed function $\mathcal{P}_{K} \widehat{v}$ in general is not contained in $X^{N}(K)$. Due to this fact, the local velocity space in [1,10] has been defined as the image of the space $\left(\mathbb{Q}_{r_{K}}(\widehat{K}) \cap H_{0}^{1}(\widehat{K})\right)^{d}$ under the operator $\mathcal{P}_{K}$. However, we will not use this modification of the internal finite element basis functions since it complicates the implementation, in particular for the derivatives of the velocity.

The idea to overcome this problem is to use a modified Piola transformation which is close to the original one. For an element $K \in \mathcal{T}_{h}$ and a given function $\widehat{v} \in\left(H^{1}(\widehat{K})\right)^{d}$, we define the modified Piola transformation $\widetilde{\mathcal{P}}_{K} \widehat{v} \in\left(H^{1}(K)\right)^{d}$ by

$$
\left(\widetilde{\mathcal{P}}_{K} \widehat{v}\right)(x):=\frac{1}{\operatorname{det}\left(B_{K}\right)} B_{K} \widehat{v}\left(F_{K}^{-1}(x)\right) \quad \forall x \in K,
$$

where $B_{K}:=D F_{K}(0)$. Then, one easily verifies that $\widetilde{\mathcal{P}}_{K} \widehat{v} \in X^{N}(K)$ for all functions $\widehat{v} \in\left(\mathbb{Q}_{r_{K}}(\widehat{K}) \cap H_{0}^{1}(\widehat{K})\right)^{d}$. Moreover, we can prove the following results.

Lemma 7. Let $\left\{\mathcal{T}_{h}\right\}$ be a family of shape-regular and 1-irregular multilevel adaptive grids generated from a coarse grid $\mathcal{T}^{0}$. Then, for all functions $\widehat{v} \in\left(H_{0}^{1}(\widehat{K})\right)^{d}$ and elements $K \in \mathcal{T}_{h}$, the following estimates are satisfied

$$
\begin{aligned}
\left|\widetilde{\mathcal{P}}_{K} \widehat{v}\right|_{1, K} & \leq C_{3} h_{K}^{-d / 2}|\widehat{v}|_{1, \widehat{K}}, \\
|\widehat{v}|_{1, \widehat{K}} & \leq C h_{K}^{d / 2}\left|\widetilde{\mathcal{P}}_{K} \widehat{v}\right|_{1, K}, \\
\left|\mathcal{P}_{K} \widehat{v}-\widetilde{\mathcal{P}}_{K} \widehat{v}\right|_{1, K} & \leq C_{4}\left(\mathcal{T}^{0}\right) h_{K}\left|\widetilde{\mathcal{P}}_{K} \widehat{v}\right|_{1, K},
\end{aligned}
$$

where the constants are independent of $\widehat{v}$ and the local mesh size $h_{K}$.

Proof. From the definition (2.7) of the modified Piola transformation and the estimates (1.6) and (1.5) it follows

$$
\left|\widetilde{\mathcal{P}}_{K} \widehat{v}\right|_{1, K} \leq C h_{K}^{1-d}\left|\widehat{v} \circ F_{K}^{-1}\right|_{1, K}
$$

Furthermore, we have

$$
\left|\widehat{v} \circ F_{K}^{-1}\right|_{1, K} \leq C\left|\operatorname{det}\left(D F_{K}\right)\right|_{0, \infty, \widehat{K}}^{1 / 2}\left|F_{K}^{-1}\right|_{1, \infty, K}|\widehat{v}|_{1, \widehat{K}} \leq C h_{K}^{d / 2-1}|\widehat{v}|_{1, \widehat{K}},
$$


which yields the estimate (2.8). From

$$
\widehat{v}(\widehat{x})=\operatorname{det}\left(B_{K}\right) B_{K}^{-1}\left(\widetilde{\mathcal{P}}_{K} \widehat{v}\right)\left(F_{K}(\widehat{x})\right)
$$

we get by means of (1.6) and (1.5) the estimates

$$
|\widehat{v}|_{1, \widehat{K}} \leq C h_{K}^{d-1}\left|\left(\widetilde{\mathcal{P}}_{K} \widehat{v}\right) \circ F_{K}\right|_{1, \widehat{K}} \leq C h_{K}^{d-1}\left|\operatorname{det}\left(D F_{K}^{-1}\right)\right|_{0, \infty, K}^{1 / 2}\left|F_{K}\right|_{1, \infty, \widehat{K}}\left|\widetilde{\mathcal{P}}_{K} \widehat{v}\right|_{1, K}
$$

which imply (2.9).

To prove $(2.10)$ we start with the splitting

$$
\mathcal{P}_{K} \widehat{v}-\widetilde{\mathcal{P}}_{K} \widehat{v}=\underbrace{\left\{\frac{1}{\operatorname{det}\left(J_{K}\right)}-\frac{1}{\operatorname{det}\left(B_{K}\right)}\right\} B_{K}\left(\widehat{v} \circ F_{K}^{-1}\right)}_{=: T_{1}}+\underbrace{\frac{1}{\operatorname{det}\left(J_{K}\right)}\left\{J_{K}-B_{K}\right\}\left(\widehat{v} \circ F_{K}^{-1}\right)}_{=: T_{2}} .
$$

Let us define the function $g_{K}: \widehat{K} \rightarrow \mathbb{R}$ as

$$
g_{K}:=\frac{1}{\operatorname{det}\left(J_{K}\right)}-\frac{1}{\operatorname{det}\left(B_{K}\right)} .
$$

Using (1.5) with $\widehat{x}=0$ and (2.11), we get

$$
\left|T_{1}\right|_{1, K} \leq C\left\|g_{K}\right\|_{0, \infty, \widehat{K}} h_{K}^{d / 2}|\widehat{v}|_{1, \widehat{K}}+C\left|g_{K}\right|_{1, \infty, \widehat{K}}\left|F_{K}^{-1}\right|_{1, \infty, K} h_{K}^{d / 2+1}\|\widehat{v}\|_{0, \widehat{K}}
$$

If we apply Friedrichs inequality and the estimate $\left|F_{K}^{-1}\right|_{1, \infty, K} \leq C h_{K}^{-1}$, which follows from (1.5) and (1.6), we conclude

$$
\left|T_{1}\right|_{1, K} \leq C\left\{\left\|g_{K}\right\|_{0, \infty, \widehat{K}}+\left|g_{K}\right|_{1, \infty, \widehat{K}}\right\} h_{K}^{d / 2}|\widehat{v}|_{1, \widehat{K}}
$$

For the function $g_{K}$ we have

$$
\begin{aligned}
\left|g_{K}\right|_{1, \infty, \widehat{K}}=\left|\frac{1}{\operatorname{det}\left(J_{K}\right)}\right|_{1, \infty, \widehat{K}} & \leq C\left|\frac{1}{\left(\operatorname{det}\left(J_{K}\right)\right)^{2}}\right|_{0, \infty, \widehat{K}}\left|\operatorname{det}\left(J_{K}\right)\right|_{1, \infty, \widehat{K}} \\
& \leq C h_{K}^{-2 d}\left|F_{K}\right|_{2, \infty, \widehat{K}}\left|F_{K}\right|_{1, \infty, \widehat{K}}^{d-1} .
\end{aligned}
$$

Using the estimate (1.8) for the multilevel adaptive grid $\mathcal{T}_{h}$, we obtain

$$
\left|g_{K}\right|_{1, \infty, \widehat{K}} \leq C\left(\mathcal{T}^{0}\right) h_{K}^{1-d}
$$

From $g_{K}(0)=0$ we get

which yields with $(2.13)$

$$
\left\|g_{K}\right\|_{0, \infty, \widehat{K}} \leq \operatorname{diam}(\widehat{K})\left|g_{K}\right|_{1, \infty, \widehat{K}}
$$

$$
\left\|g_{K}\right\|_{0, \infty, \widehat{K}} \leq C\left(\mathcal{T}^{0}\right) h_{K}^{1-d}
$$

If we combine (2.12) with (2.14) and (2.13) we get the estimate

$$
\left|T_{1}\right|_{1, K} \leq C\left(\mathcal{T}^{0}\right) h_{K}^{1-d / 2}|\widehat{v}|_{1, \widehat{K}}
$$

For the estimation of the term $T_{2}$, we introduce the functions $\phi_{K}: \widehat{K} \rightarrow \mathbb{R}$ and $G_{K}: \widehat{K} \rightarrow \mathbb{R}^{d \times d}$ defined as

$$
\phi_{K}(\widehat{x}):=\frac{1}{\operatorname{det}\left(J_{K}(\widehat{x})\right)}, \quad G_{K}(\widehat{x}):=J_{K}(\widehat{x})-B_{K} .
$$


Now, $T_{2}$ can be estimated as follows

$$
\begin{aligned}
\left|T_{2}\right|_{1, K} \leq & C h_{K}^{d / 2}\left\{\left|\phi_{K}\right|_{1, \infty, \widehat{K}}\left\|G_{K}\right\|_{0, \infty, \widehat{K}}\|\widehat{v}\|_{0, \widehat{K}}+\left\|\phi_{K}\right\|_{0, \infty, \widehat{K}}\left|G_{K}\right|_{1, \infty, \widehat{K}}\|\widehat{v}\|_{0, \widehat{K}}\right. \\
& \left.+\left\|\phi_{K}\right\|_{0, \infty, \widehat{K}}\left\|G_{K}\right\|_{0, \infty, \widehat{K}}|\widehat{v}|_{1, \widehat{K}}\right\}\left|F_{K}^{-1}\right|_{1, \infty, K}
\end{aligned}
$$

where the norm and semi norm of $G_{K}$ are defined by

$$
\left\|G_{K}\right\|_{0, \infty, \widehat{K}}:=\sup _{\widehat{x} \in \widehat{K}}\left\|G_{K}(\widehat{x})\right\|_{\infty}, \quad\left|G_{K}\right|_{1, \infty, \widehat{K}}:=\max _{j=1, \ldots, d} \sup _{\widehat{x} \in \widehat{K}}\left\|\frac{\partial G_{K}}{\partial \widehat{x}_{j}}(\widehat{x})\right\|_{\infty} .
$$

For the function $\phi_{K}$, we have by (1.6) and (2.13)

$$
\left\|\phi_{K}\right\|_{0, \infty, \widehat{K}} \leq C h_{K}^{-d}, \quad\left|\phi_{K}\right|_{1, \infty, \widehat{K}}=\left|g_{K}\right|_{1, \infty, \widehat{K}} \leq C\left(\mathcal{T}^{0}\right) h_{K}^{1-d} .
$$

Using (1.9) and (1.8), the matrix function $G_{K}$ can be estimated as follows

$$
\left\|G_{K}\right\|_{0, \infty, \widehat{K}} \leq C_{2}\left(\mathcal{T}^{0}\right) h_{K}^{2}, \quad\left|G_{K}\right|_{1, \infty, \widehat{K}} \leq C\left|F_{K}\right|_{2, \infty, \widehat{K}} \leq C\left(\mathcal{T}^{0}\right) h_{K}^{2}
$$

If we apply Friedrichs inequality and the estimate $\left|F_{K}^{-1}\right|_{1, \infty, K} \leq C h_{K}^{-1}$, we obtain from (2.16)

$$
\begin{aligned}
\left|T_{2}\right|_{1, K} & \leq C\left(\mathcal{T}^{0}\right) h_{K}^{d / 2}\left\{h_{K}^{1-d} h_{K}^{2}+h_{K}^{-d} h_{K}^{2}+h_{K}^{-d} h_{K}^{2}\right\} h_{K}^{-1}|\widehat{v}|_{1, \widehat{K}} \\
& \leq C\left(\mathcal{T}^{0}\right) h_{K}^{1-d / 2}|\widehat{v}|_{1, \widehat{K}}
\end{aligned}
$$

Now, the assertion (2.10) follows from (2.15), (2.17) and (2.9).

\subsection{Proof of the local inf-sup condition}

For our pair of finite element spaces, the following local inf-sup condition is known.

Lemma 8. Let $\mathcal{T}_{h}$ be a shape-regular grid. Then, for each element $K \in \mathcal{T}_{h}$, the pair $\left(X^{N}(K), M^{N}(K)\right)$ of the mapped local finite element spaces defined in (2.1) and (2.2), satisfies the local inf-sup condition (2.3) with a constant $\beta_{0}=\lambda\left(r_{K}\right)>0$ which depends on the local degree $r_{K}$ but which is independent of $K$ and the mesh size $h$.

Proof. See the proof of Theorem 8 in [20].

This result implies a local inf-sup condition (2.3) with a constant

$$
\beta_{0}=\beta_{0}^{*}\left(r^{*}\right):=\min _{r=1, \ldots, r^{*}} \lambda(r) \quad \text { where } \quad r^{*}:=\max _{K \in \mathcal{T}_{h}} r_{K}
$$

However, the dependence of $\beta_{0}^{*}\left(r^{*}\right)$ on the maximum polynomial degree $r^{*}$ has not been investigated in [20]. For a grid $\mathcal{T}_{h}$ consisting of affine equivalent elements, the estimate $\beta_{0}^{*}\left(r^{*}\right) \geq C\left(r^{*}\right)^{-(d-1) / 2}$ has been shown in [24]. In the following, we will prove that, for a sufficiently small mesh size, the local inf-sup condition (2.3) holds with a constant $\beta_{0}$ which is independent of the mesh size $h$ and the polynomial degree vector $\underline{r}$.

A main ingredient of our analysis is the following result of Bernardi and Maday which we will apply on the reference element. 
Lemma 9. For $\widehat{K}=(-1,1)^{d}$ and an arbitrary integer $r \geq 2$, let $\widehat{X}_{r}:=\left(\mathbb{Q}_{r}(\widehat{K}) \cap H_{0}^{1}(\widehat{K})\right)^{d}$ and $\widehat{M}_{r}:=$ $\mathbb{P}_{r-1}(\widehat{K}) \cap L_{0}^{2}(\widehat{K})$. Then, it holds

$$
\forall \widehat{q} \in \widehat{M}_{r} \quad \exists \widehat{v} \in \widehat{X}_{r}: \quad(\widehat{\operatorname{div}} \widehat{v}, \widehat{q})_{\widehat{K}} \geq \widehat{\beta}_{0}|\widehat{v}|_{1, \widehat{K}}\|\widehat{q}\|_{0, \widehat{K}}
$$

with a constant $\widehat{\beta}_{0}>0$ which is independent of the polynomial degree $r$.

Proof. See [4].

Now, using the original and modified Piola transformation, the estimate (2.19) can be transformed to the pair of the local finite element spaces $\left(X^{N}(K), M^{N}(K)\right)$ defined in (2.1) and (2.2).

Lemma 10. Let $\left\{\mathcal{T}_{h}\right\}$ be a family of shape-regular and 1-irregular multilevel adaptive grids generated from a coarse grid $\mathcal{T}^{0}$. Then, there exists a sufficiently small mesh size $h_{0}=h_{0}\left(\mathcal{T}^{0}\right)$ such that for all grids $\mathcal{T}_{h}$ with $h \leq h_{0}$ and all $K \in \mathcal{T}_{h}$ it holds

$$
\forall q \in M^{N}(K) \quad \exists v \in X^{N}(K): \quad(\operatorname{div} v, q)_{K} \geq \beta_{0}|v|_{1, K}\|q\|_{0, K},
$$

with a constant $\beta_{0}>0$ which is independent of the mesh size and the polynomial degree vector $\underline{r}$. Therefore, the local inf-sup condition (2.3) is satisfied with this constant $\beta_{0}$.

Proof. For a given $q \in M^{N}(K)$, let $\widehat{q}:=q \circ F_{K} \in \widehat{M}_{r}$. Due to Lemma 9, there exists a $\widehat{v} \in \widehat{X}_{r}$ satisfying (2.19). Choose $v:=h_{K}^{d} \mathcal{P}_{K} \widehat{v}$. Then, it holds

$$
(\operatorname{div} v, q)_{K}=h_{K}^{d}(\widehat{\operatorname{div}} \widehat{v}, \widehat{q})_{\widehat{K}} \geq \widehat{\beta}_{0} h_{K}^{d}|\widehat{v}|_{1, \widehat{K}}\|\widehat{q}\|_{0, \widehat{K}} .
$$

Using a standard argument for the estimation between the norms of $\widehat{q}$ and $q$ we obtain

$$
(\operatorname{div} v, q)_{K} \geq C_{5} h_{K}^{d / 2}|\widehat{v}|_{1, \widehat{K}}\|q\|_{0, K}
$$

with a constant $C_{5}$ which is independent of the mesh size and the degree vector $\underline{r}$. Since $v$ in general is not contained in $X^{N}(K)$, we define the function $\widetilde{v}:=h_{K}^{d} \widetilde{\mathcal{P}}_{K} \widehat{v}$ which satisfies $\widetilde{v} \in X^{N}(K)$. From Lemma 7 we get

$$
\begin{aligned}
|\widehat{v}|_{1, \widehat{K}} & \geq C_{3}^{-1} h_{K}^{-d / 2}|\widetilde{v}|_{1, K}, \\
\left|(\operatorname{div}(\widetilde{v}-v), q)_{K}\right| & \leq C_{4}\left(\mathcal{T}^{0}\right) \sqrt{d} h_{K}|\widetilde{v}|_{1, K}\|q\|_{0, K} .
\end{aligned}
$$

Using (2.21)-(2.23), this implies

$$
\begin{aligned}
(\operatorname{div} \widetilde{v}, q)_{K} & =(\operatorname{div} v, q)_{K}+(\operatorname{div}(\widetilde{v}-v), q)_{K} \\
& \geq C_{5} h_{K}^{d / 2}|\widehat{v}|_{1, K}\|q\|_{0, K}-C_{4}\left(\mathcal{T}^{0}\right) \sqrt{d} h_{K}|\widetilde{v}|_{1, K}\|q\|_{0, K} \\
& \geq\left(\frac{C_{5}}{C_{3}}-C_{4}\left(\mathcal{T}^{0}\right) \sqrt{d} h_{K}\right)|\widetilde{v}|_{1, K}\|q\|_{0, K} \\
& \geq \beta_{0}|\widetilde{v}|_{1, K}\|q\|_{0, K}
\end{aligned}
$$

for all $K \in \mathcal{T}_{h}$ and a grid $\mathcal{T}_{h}$ with $h \leq h_{0}:=C_{5} /\left(2 C_{3} C_{4}\left(\mathcal{T}^{0}\right) \sqrt{d}\right)$ where $\beta_{0}=C_{5} /\left(2 C_{3}\right)$. Therefore, (2.20) is proved since $\widetilde{v} \in X^{N}(K)$. 


\subsection{Summary}

Now, we can state the main result on the discrete inf-sup condition (0.2) for our finite element spaces.

Theorem 11. Let $\left\{\mathcal{T}_{h}\right\}$ be a family of shape-regular and 1-irregular multilevel adaptive grids generated from a coarse grid $\mathcal{T}^{0}$. Then, there exists a sufficiently small mesh size $h_{0}=h_{0}\left(\mathcal{T}^{0}\right)$ such that the following result holds. For the pair of finite element spaces $\left(X^{N}, M^{N}\right)$, defined in (1.11) and (1.12) with $X^{N}=\left(S^{N}\right)^{d}$ and the degree vector $\underline{r}$, the global inf-sup condition $(0.2)$ is fulfilled with a constant $\beta>0$ that satisfies the following estimates

$$
\beta \geq \begin{cases}\beta^{*}\left(r^{*}\right), & \forall \mathcal{T}_{h}, \underline{r} \text { with } \max _{K \in \mathcal{T}_{h}} r_{K} \leq r^{*}, \\ \beta_{1}, & \forall \mathcal{T}_{h}, \underline{r} \text { with } h \leq h_{0},\end{cases}
$$

where $\beta_{1}$ is a positive constant which is independent of the mesh size $h$ and the degree vector $\underline{r}$.

Proof. Due to Lemma 4, it is sufficient to verify the global and local inf-sup condition. The global inf-sup condition is guaranteed by Lemma 5. Then, the first estimate in (2.24) is a consequence of Lemma 8 and (2.18). The second estimate in (2.24) follows from Lemma 10.

By Theorem 11, the uniform inf-sup stability of our $h p$-finite element pair on multilevel adaptive grids is guaranteed under the following $h p$-regime. For coarser grids $\mathcal{T}_{h}$ with $h>h_{0}$, we do not allow the polynomial degree to be beyond a moderate degree $r^{*}$, i.e. $\max _{K \in \mathcal{T}_{h}} r_{K} \leq r^{*}$, but for finer grids $\mathcal{T}_{h}$ with $h \leq h_{0}$ the polynomial degree can be chosen arbitrarily.

\section{REFERENCES}

[1] M. Ainsworth and P. Coggins, A uniformly stable family of mixed $h p$-finite elements with continuous pressures for incompressible flow. IMA J. Numer. Anal. 22 (2002) 307-327.

[2] D.N. Arnold, D. Boffi and R.S. Falk, Approximation by quadrilateral finite elements. Math. Comput. 71 (2002) 909-922.

[3] I. Babuška and A. Miller, A feedback finite element method with a posteriori error estimation. I. The finite element method and some basic properties of the a posteriori error estimator. Comput. Methods Appl. Mech. Engrg. 61 (1987) 1-40.

[4] C. Bernardi and Y. Maday, Uniform inf-sup conditions for the spectral discretization of the Stokes problem. Math. Models Methods Appl. Sci. 9 (1999) 395-414.

[5] D. Boffi and L. Gastaldi, On the quadrilateral $\mathrm{Q}_{2}-\mathrm{P}_{1}$ element for the Stokes problem. Int. J. Numer. Methods Fluids 39 (2002) $1001-1011$.

[6] J.M. Boland and R.A. Nicolaides, Stability of finite elements under divergence constraints. SIAM J. Numer. Anal. 20 (1983) $722-731$.

[7] S. Bönisch, V. Heuveline and P. Wittwer, Adaptive boundary conditions for exterior flow problems. J. Math. Fluid Mech. 7 (2005) 85-107.

[8] F. Brezzi and R.S. Falk, Stability of higher-order Hood-Taylor methods. SIAM J. Numer. Anal. 28 (1991) 581-590.

[9] F. Brezzi and M. Fortin, Mixed and Hybrid Finite Element Methods, Springer Series in Computational Mathematics 15. Springer-Verlag (1991).

[10] L. Chilton and M. Suri, On the construction of stable curvilinear $p$ version elements for mixed formulations of elasticity and Stokes flow. Numer. Math. 86 (2000) 29-48.

[11] V. Girault and P.-A. Raviart, Finite Element Methods for Navier-Stokes equations. Springer-Verlag, Berlin-HeidelbergNew York (1986).

[12] V. Heuveline and M. Hinze, Adjoint-based adaptive time-stepping for partial differential equations using proper orthogonal decomposition. Technical report, University Heidelberg, Germany, SFB 359 (2004).

[13] V. Heuveline and R. Rannacher, A posteriori error control for finite element approximations of elliptic eigenvalue problems. Adv. Comput. Math. 15 (2001) 107-138.

[14] V. Heuveline and R. Rannacher, Duality-based adaptivity in the hp-finite element method. J. Numer. Math. 11 (2003) 95-113.

[15] V. Heuveline and F. Schieweck, $H^{1}$-interpolation on quadrilateral and hexahedral meshes with hanging nodes. Computing 80 (2007) 203-220.

[16] V. Heuveline and F. Schieweck, On the inf-sup condition for higher order mixed fem on meshes with hanging nodes. ESAIM: M2AN 41 (2007) 1-20.

[17] G. Matthies, Mapped finite elements on hexahedra. Necessary and sufficient conditions for optimal interpolation errors. Numer. Algorithms 27 (2001) 317-327.

[18] G. Matthies, Finite element methods for free boundary value problems with capillary surfaces. Ph.D. thesis, Fakultät für Mathematik, Otto-von-Guericke-Universität Magdeburg, Germany (2002). [Published at Shaker-Verlag Aachen]. 
[19] G. Matthies and F. Schieweck, On the reference mapping for quadrilateral and hexahedral finite elements on multilevel adaptive grids. Computing 80 (2007) 95-119.

[20] G. Matthies and L. Tobiska, The inf-sup condition for the mapped $Q_{k}-P_{k-1}^{\text {disc }}$ element in arbitrary space dimensions. Computing 69 (2002) 119-139.

[21] S. Schötzau, C. Schwab and R. Stenberg, Mixed $h p$-fem on anisotropic meshes II: Hanging nodes and tensor products of boundary layer meshes. Numer. Math. 83 (1999) 667-697.

[22] C. Schwab, $p$ - and hp-Finite Element Methods, Theory and Applications in Solid and Fluid Mechanics, Numerical Mathematics and Scientific Computation. Oxford Science Publications, Clarendon Press (1998).

[23] R. Stenberg, Error analysis of some finite element methods for the Stokes problem. Math. Comput. 54 (1990) 495-508.

[24] R. Stenberg and M. Suri, Mixed $h p$ finite element methods for problems in elasticity and Stokes flow. Numer. Math. 72 (1996) $367-389$.

[25] A. Toselli and C. Schwab, Mixed $h p$-finite element approximations on geometric edge and boundary layer meshes in three dimensions. Numer. Math. 94 (2003) 771-801. 